\title{
Analysis of Transmission Delay of Wireless Sensor Network Based on GNSS Signal
}

\author{
LIU Rong ${ }^{1}$, XUE Fang ${ }^{2}$ \\ \{wz26677@163.com ${ }^{1}$, aaa21152@163.com²\} \\ ${ }^{1}$ School of Information Engineering, Changsha Medical University, Changsha 410219, China; \\ ${ }^{2}$ Information Technology Center, Jimei University, XiaMen 361021, China
}

\begin{abstract}
Aiming at the problem that the traditional network transmission delay analysis method occupies too much memory of the operating platform during operation, a GNSS (Global Navigation Satellite System) signal-based transmission delay analysis method for wireless sensor networks is proposed. Establish a cluster structure and use the method based on network fusion and clustering algorithms to perceive the transmission delay of wireless sensor networks. Delay parameterized processing of GNSS signals, and the establishment of a wireless sensor network data transmission model, complete the study of wireless sensor network transmission delay analysis methods. The comparison experiment between the design and the traditional delay analysis method proves that the GNSS signal-based delay analysis method occupies less memory of the operating platform and performs better under the same task.
\end{abstract}

Keywords: GNSS signal; Wireless sensor network; Transmission delay; Analytical method

\section{1 introduction}

GNSS is the unified name for single satellite navigation and positioning of Beidou system, GPS (Global Positioning System), GLONASS (Global Navigation Satellite System), and Galileo system. It can also refer to the enhanced system of single satellite navigation and positioning, and also refers to the addition of all satellite navigation and positioning and its enhanced system. GNSS is a large system composed of multiple satellite navigation and positioning systems and their enhanced systems[1]. GNSS uses satellites as star-level radio navigation systems for navigation stations, and provides all-weather, high-precision position, speed, and time information for all types of military and civilian carriers on land, sea, air, and sky.With the progress and development of GNSS technology of global satellite 
navigation system, the application fields of the system are becoming more and more extensive, especially in navigation and positioning and atmospheric measurement.

A wireless sensor network is a distributed sensor network. Its tip is a sensor that can sense and check the outside world. The sensor in wireless sensor network is wireless communication, and the network setting is flexible. It can change the location of the device at any time, or connect to the Internet by wire or wireless, and form a multi hop self-organized network through wireless communication [2].There are two types of nodes in wireless sensor networks, one is the convergence node and the other is the sensor node. The convergence node mainly refers to the gateway's ability to remove false report data from the sensor nodes and combine it with related reports to fuse the data and judge the events that occur.The connection between the sink node and the user node can be directly communicated through a wide area network or satellite, and the collected data is processed [3]. Due to the placement of nodes in a wireless sensor network, there is a certain transmission delay between the networks. Network transmission delay is a phenomenon in which certain steps take longer than normal transmission time when data is transmitted in the network. Literature [4] method takes up too many resources of the operating platform when analyzing the network with too many nodes, which has great limitations, resulting in too much memory of the operating platform. To solve this problem, this paper proposes a transmission delay analysis method based on GNSS signal in wireless sensor network.

\section{Analysis method of transmission delay of wireless sensor network based on GNSS signal}

\subsection{Wireless sensor network transmission delay perception}

In order to analyze the transmission delay of wireless sensor networks and reduce the energy consumption of wireless sensor networks, this paper uses a method based on network fusion and clustering algorithm to detect the transmission delay of wireless sensor networks.

First, establish the cluster structure shown in the following figure. Nodes A and $\mathrm{B}$ are cluster members and node $\mathrm{C}$ is the cluster head. 


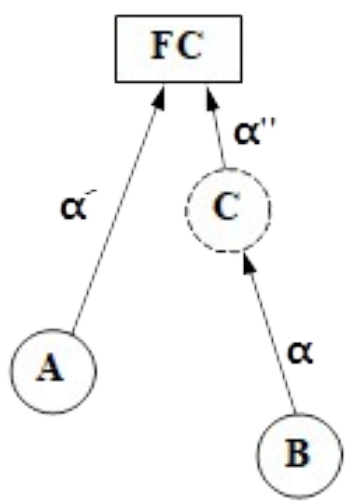

Fig. 1 Schematic diagram of cluster structure

A and B nodes transmit data packet $\alpha$ to cluster head C. Assume that the unit time required to transmit data $\alpha$ from one node to another node is $t_{T X}$, and the corresponding transmitter and receiver energy consumption is $e_{T X}$ and $e_{R X}$, respectively.To simplify the calculation process, energy consumption is ignored during data fusion. At the same time, the fusion center (FC) can be regarded as a device node with sufficient energy.If nodes $\mathrm{A}, \mathrm{B}$ and $\mathrm{C}$ can be fused into a single data packet size $\alpha^{\prime}\left(\alpha^{\prime}=\alpha\right)$, the total delay $\mathrm{M}$ and $\mathrm{N}$ is the smallest.

When the output data $\alpha^{\prime}>\alpha$, the delay and energy consumption of the wireless sensor network will increase. The calculation formula is as follows:

$$
\left\{\begin{array}{l}
t_{T O T}=2 t_{T X}+\frac{\alpha^{\prime}}{\alpha} t_{T X} \\
e_{T O T}=2\left(e_{T X}+e_{R X}\right)+\frac{\alpha^{\prime}}{\alpha} e_{T X}
\end{array}\right.
$$

According to the above analysis process, assuming that there are $N$ wireless sensor nodes in the wireless sensor network, a network with $\mathrm{k}$ clusters will be constructed. According to the basic properties of the tree structure shown in Figure 1, there are: 


$$
\sum_{i=1}^{k-1} m_{i}<N \leq \sum_{i=1}^{k} m_{i}{ }^{(2)}
$$

In formula (2), $m_{i}$ is the largest number of nodes in the $i^{\text {-th cluster, and can be }}$ expressed as:

$$
m_{i}=2 m_{i-1}, m_{i}=1^{(3)}
$$

$m_{i}=2^{i}-1$ can be obtained using the recursive formula. Then formula (2) can be converted into the following form:

$$
2^{k-1}-1<N \leq 2^{k}-1^{(4)}
$$

Analysis of the cluster structure shown in Figure 1 shows that smaller clusters can shorten the data aggregation process to reduce the delay time [5]. Therefore, before the $k$-th cluster is filled with cluster members, the $k-1$-th cluster has been filled with cluster members. As can be seen from formula (4), taking the number of cluster structure nodes $N=7$ as an example, $\mathrm{k}=3$ clusters are constructed. This structure is a multilayer cluster structure constructed by multiple clusters, that is, a multi-hop structure exists in one cluster. Multi-hop may cause additional data fusion delay and network energy consumption.

Therefore, the aggregation structure shown in the figure below is improved, in which the virtual circle represents the cluster head node, the solid circle represents the cluster member node, FC represents the center node of cluster structure fusion, and the number represents the interleaving communication sequence of cluster head node and FC.

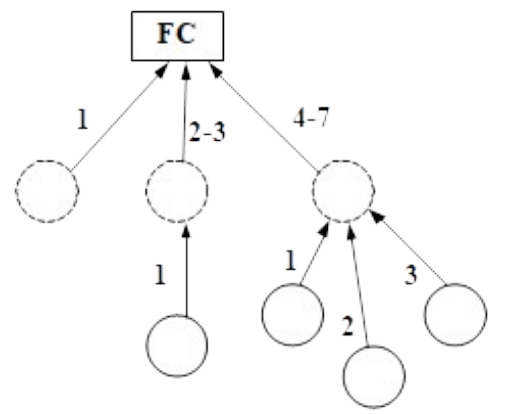


Fig.2 Data aggregation structure when $\mathrm{N}=7$ after improvement

As can be seen from Figure 2, the improved structure has multiple clusters consisting of multiple single-hop cluster structures of different sizes, that is, there is no multi-hop structure in a cluster [6].Based on the above analysis process, it can be determined that before the larger cluster is ready, the smaller cluster can complete communication with the FC. Therefore, the delay time of the entire wireless sensor network is determined by its largest cluster.Therefore, a clustering algorithm is used to cluster nodes in a wireless sensor network. When the largest cluster after clustering is determined, the perception of the transmission delay of the wireless sensor network is completed. When the transmission delay is perceived in the wireless sensor network, the delay parameter haul processing is performed on the GNSS signal.

\subsection{GNSS signal delay parameter processing}

When electromagnetic waves pass through the air, there will be a certain degree of refraction. Not only will the speed change, but the propagation path will also change due to bending. The propagation velocity $v$ of the electromagnetic wave in the medium can be expressed as:

$$
v=\frac{c}{n}^{(5)}
$$

In formula (5), $c$ is the speed of light in a vacuum, and the unit is $\mathrm{m} / \mathrm{s} ; n$ is the refractive index of the medium. When the GNSS signal propagates in the air, the ranging code propagates at the group velocity $v_{g}$, and the carrier phase propagates at the phase velocity $v_{p}$.

When the signal frequency is $f$ and the wavelength is $\lambda$, the phase velocity $v_{p}$ and group velocity $v_{g}$ can be defined according to the following formula.

$$
\left\{\begin{array}{l}
v_{p}=f \times \lambda \\
v_{g}=-\frac{d f}{d \lambda} \times \lambda^{2}
\end{array}\right.
$$


According to the well-known Appleton-Hartree formula, when electromagnetic waves propagate in wireless sensor networks, assuming that ionic collision and thermal motion are not considered, the phase refractive index can be expressed according to the following formula [7].

$$
n_{p}^{2}=1-\frac{X}{1-\frac{Y_{T}^{2}}{2(1-X)} \pm \sqrt{Y_{L}^{2}+\frac{Y_{T}^{2}}{4(1-X)^{2}}}} \text { (7) }
$$

In formula (7), it is the displacement transmitted in the horizontal direction in the wireless sensor network and the displacement transmitted in the vertical direction in the wireless sensor network. The calculation formula of the above three parameters is as follows:

$$
\left\{\begin{array}{l}
X=\frac{e^{2} N_{e}}{4 \pi^{2} \varepsilon_{0} m f} \\
Y_{T}=\frac{\mu_{0} e\left(H_{0} \sin \theta\right)}{2 \pi m f}(8) \\
Y_{L}=\frac{\mu_{0} e\left(H_{0} \cos \theta\right)}{2 \pi m f}
\end{array}\right.
$$

In the above formula, $e$ is the amount of charge carried by the electron, and the value of $e^{\text {is }} e=1.6022 \times 10^{-19} C ; \quad N_{e}$ is the electron density of the electromagnetic wave in the wireless sensor network, and its unit is / $\mathrm{m} 3$; $\varepsilon_{0}$ is the dielectric constant of the electromagnetic wave in the air; $m$ is the mass of the electron, and the value of $m$ is $m=9.1096 \times 10^{-31} \mathrm{~kg} ; f$ is the frequency of the electromagnetic wave signal; $\mu_{0}$ is the magnetic permeability $\mu_{0}=4 \pi \times 10^{-7} \mathrm{H} / \mathrm{m}$ of the electromagnetic wave in the vacuum; $H_{0}$ is the magnetic field strength of the geomagnetic field; $\theta$ is the angle between the direction of the geomagnetic field and the direction of propagation of the electromagnetic wave signal [8].The above 
analysis process is mathematically processed, and a physical quantity $T_{C}$ is introduced, where $T_{C}$ represents the total electron content of the GNSS signal transmitted in the wireless sensor network. The calculation formula is as follows:

$$
T_{C}=\int_{S} N_{e} d S(9)
$$

The unit of the physical quantity $T_{C}$ is unit / $\mathrm{m} 2$. The meaning of $T_{C}$ is the unit area as the base area. After the above processing, the parameter delay processing of GNSS signal transmission in wireless sensor network is completed. In order to analyze the transmission delay of wireless sensor networks, based on the above research, a data transmission model of wireless sensor networks based on Markov's principle is established.

\subsection{Building a data transmission model for wireless sensor networks}

According to Markov's principle, let $P$ be a matrix composed of transition probabilities $P_{i j}$ and state space $I=\{1,2, \cdots\}$, then use the following formula to represent the transition probability matrix of the system state.

$$
\begin{aligned}
& P=\left[\begin{array}{cccc}
P_{11} & P_{12} & \cdots & P_{1 n} \\
P_{21} & P_{22} & \cdots & P_{2 n} \\
\vdots & \vdots & \ddots & \vdots \\
P_{n 1} & P_{n 2} & \cdots & P_{n n}
\end{array}\right](10) \\
& P_{i j} \geq 0, i, j=1,2, \cdots, N \\
& \sum_{j=1}^{N} P_{i j}=1, i=1,2, \cdots, N
\end{aligned}
$$

In the above formula, $P_{i j}$ is the conditional probability that the communication transmission state of the wireless sensor network changes from the $i^{\text {-state to the }} j^{-}$ state. In the wireless sensor network, channel transfer occurs during transmission. According to the Markov model, the wireless sensor network is divided into channels 
according to the signal-to-noise ratio of the transmitted signal. The signal-to-noise ratio $\gamma$ is divided into $K+1$ parts, and the segmentation point of the signal-tonoise ratio division is $\left\{\gamma_{k}\right\}_{k=0}^{k=K+1}$. When $\gamma \subset\left\{\gamma_{k}\right\}_{k=0}^{k=K+1}$, this channel belongs to the state $k$, so the channel has a total of $K$ states.To avoid deep fading, it is assumed that no transmission occurs during state $\mathrm{k}$, and the rate at this time is $R_{0}=0$ bit / symbol.According to the principle of Markov model, when the wireless sensor network channel is divided, the signal-to-noise ratio $\gamma$ is used as the threshold value and divided into several channel states. Different channel states correspond to their corresponding transmission modes, and the transition between channel states is achieved by transition probability.

Therefore, the threshold $\left\{\gamma_{k}\right\}_{k=0}^{k=K+1}$ of the SNR division interval can be obtained according to the following calculation process:

Let $k=K, \gamma_{k+1}=+\infty$, for each state ${ }_{n}$ of the wireless sensor network, find a unique $\gamma_{k} \subset\left[0, \gamma_{k+1}\right]$, and $\gamma_{k}$ satisfies $\frac{P E R_{k}}{P P_{0}}$. Among them, $P_{0}$ is the probability of data packet transmission error, and $\overline{P E R}_{k}$ is the average error probability of data packet transmission when the wireless sensor network is in a channel state. $\overline{P E R}_{k}$ is related to the average signal-to-noise ratio, channel state parameters and channel state received by the receiving nodes in the wireless sensor network.

At that time $k>1$, ordered $k=k-1$, continue to look for the only $\gamma_{k} \subset\left[0, \gamma_{k+1}\right]^{\text {;If }} k \leq 1$, let $\gamma_{0}=0$, when the signal-to-noise ratio of the GNSS signal transmitted by the wireless sensor network is within the $\left[\gamma_{k}, \gamma_{k+1}\right)$ interval, the corresponding channel state is, and the signal-to-noise ratio at this time is the 
threshold value when the channel is divided[10-13].

Establish a Markov transmission model of various channel states as shown in the figure below.

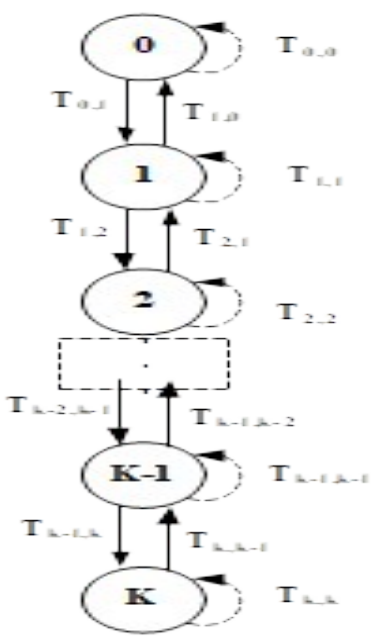

Figure 3 Data transmission model of wireless sensor network

In the figure above, $T_{k, k^{\prime}}$ represents the transition probability between channels. Under slow fading channel conditions, channel state transitions only occur between adjacent channels, and the transition probability between non-adjacent channels is $T_{k, k^{\prime}}=0,\left|k-k^{\prime}\right| \geq 2$. The transition probability of adjacent states can be calculated according to the following formula.

$$
\left\{\begin{array}{l}
T_{k, k+1}=\frac{N_{k+1} T_{f}}{P(k)_{r}}, k=0,1,2, \cdots, K-1 \\
T_{k, k-1}=\frac{N_{k} T_{f}}{P(k)_{r}}, k=1,2, \cdots, K
\end{array}\right.
$$

In formula (11), $T_{f}$ represents the transmission time of a data packet, and $P(k)_{r}$ is the probability of an error in data packet transmission. The probability that 
the channel state does not change in adjacent time slots can be calculated according to the following formula:

$$
T_{k, k}=\left\{\begin{array}{l}
1-T_{k, k+1}-T_{k, k-1}, 0<k<K \\
1-T_{0,1}, k=0 \\
1-T_{k, k-1}, k=K
\end{array}\right.
$$

According to the above calculation process, the transition probability of adjacent states can be calculated. This can determine the transition probability matrix of the wireless network sensor network, complete the construction of the wireless sensor network transmission model, and analyze the wireless sensor network transmission delay according to the model.

\subsection{Analyze Wireless Sensor Network Transmission Delay}

After establishing a wireless sensor network transmission model, use GNSS signals to analyze the wireless sensor network transmission delay. In the wireless sensor network transmission model, if the GNSS signal flows from the $k$-th channel $i$ in the network to the $k-1$-th channel $j$, then:

$$
\left\{\begin{array}{l}
L T_{k}(i, j)=L T_{k-1}(j)+\frac{1}{L}+W T_{k}(i, j) \\
S V_{k}(i, j)=S V_{k-1}(j)+W T_{k}(i, j)
\end{array}\right.
$$

In formula (13), $L T_{k}(i, j)$ is the delay time required to obtain data of the $k-1^{-}$ th channel $j$ from the $k^{\text {-th channel }} i ; S V_{k}(i, j)$ is the service required to obtain data of the $k-1$-th channel $j$ from the k-th channel $i$ time; $\frac{1}{L}$ is the time required to transmit a data envelope between two adjacent stages; $W T_{k}(i, j)$ is the average waiting time to obtain the $k-1$-th channel $j$ from the $k$-th channel $i$.

Since the relevant parameters of the GNSS signal are known, according to the above process, the delay time when the wireless sensor network transmits data can be determined. At present, the research of transmission delay analysis method based on GNSS signal has been completed. 


\section{Test experiment}

In this paper, a method for analyzing transmission delay of wireless sensor networks based on GNSS signals is studied. In order to verify the effectiveness of the method, an experimental procedure is designed. By analyzing the experimental data, the corresponding experimental conclusions are drawn.

\subsection{Experimental content}

This experiment is in the form of a comparative experiment. The experimental comparison group is the traditional network transmission delay analysis method. The experimental group is the GNSS signal-based wireless sensor network transmission delay analysis method studied in this paper. The comparison index of the experiment is the ratio of the memory occupied by the loading platform to the memory occupied by the two analysis methods when analyzing the same experimental network transmission delay.

The better performing transfer latency analysis model uses less running memory. In order to ensure the scientific and effective experimental data, the experimental process controls only the experimental variables, and the experiment is completed in accordance with the experimental process. Analyze experimental data and draw experimental conclusions.

\subsection{Experiment preparation and process}

The delay analysis methods of the experimental group and the comparison group were respectively loaded on the identically configured runners. Before the experiment, the two runners were tested to run well. After the two sets of analysis methods were loaded, the relevant data monitoring software could be run normally.

Set up a wireless sensor network as shown in the figure in the laboratory. 


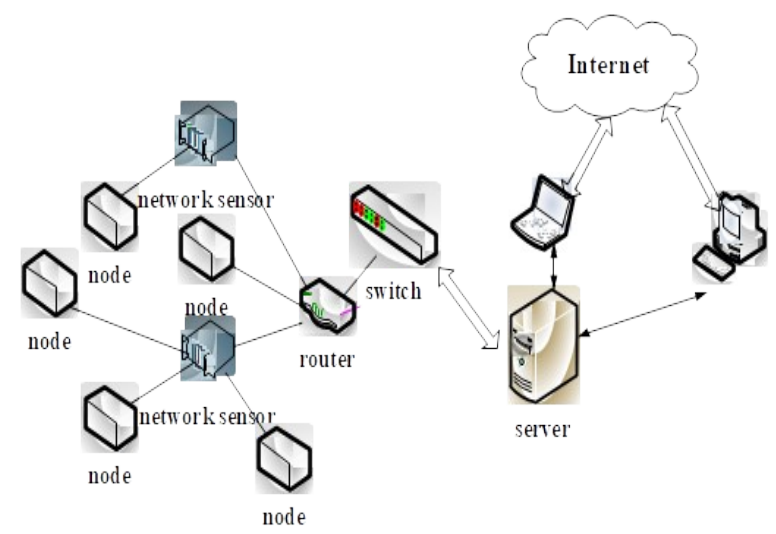

Figure 4 Experimental environment

In order to test the two analysis methods, 10 experiments were performed. During the experiment, the relevant parameters of the experimental network were set according to the following table.

Table 1 Related parameters of the experimental network

\begin{tabular}{cccc}
\hline $\begin{array}{c}\text { The serial } \\
\text { number }\end{array}$ & $\begin{array}{c}\text { Number } \\
\text { of nodes }\end{array}$ & $\begin{array}{c}\text { Transmitted } \\
\text { data } \\
\text { volume } \\
\text { /GB }\end{array}$ & $\begin{array}{c}\text { Transmission } \\
\text { distance } / \mathrm{m}\end{array}$ \\
\hline 1 & 20 & 10 & 30 \\
2 & 30 & 20 & 40 \\
3 & 45 & 50 & 60 \\
4 & 45 & 60 & 100 \\
5 & 80 & 120 & 100 \\
6 & 80 & 150 & 150 \\
7 & 100 & 185 & 150 \\
8 & 75 & 200 & 200 \\
9 & 90 & 220 & 200
\end{tabular}




\begin{tabular}{llll}
\hline 10 & 110 & 240 & 300
\end{tabular}

After setting the parameters of the experimental network, run the experimental network. Related software and equipment are used to control the network transmission delay, and the experimental group and comparative group analysis methods are used to analyze the transmission delay in the experimental network. The monitoring software of the experimental running platform occupies the platform's memory during the running of the two methods and outputs monitoring data. Analyze the experimental data and draw relevant conclusions.

\subsection{Experimental results}

The experimental results are shown in the following table. The data in the table is analyzed to obtain corresponding conclusions.

Table 2 Experimental results

\begin{tabular}{ccc}
\hline $\begin{array}{c}\text { The serial } \\
\text { number }\end{array}$ & $\begin{array}{c}\text { Experimental } \\
\text { method/\% }\end{array}$ & $\begin{array}{c}\text { Comparison } \\
\text { group } \\
\text { method/\% }\end{array}$ \\
\hline 1 & 25.8 & 49.4 \\
2 & 21.7 & 454 \\
3 & 24.5 & 52.3 \\
4 & 25.2 & 50.3 \\
5 & 25.4 & 56.3 \\
6 & 28.5 & 56.4 \\
7 & 27.5 & 50.4 \\
8 & 28.4 & 53.5 \\
9 & 24.4 & 41.2 \\
10 & 29.9 & 46.9 \\
\hline
\end{tabular}

It can be seen from the analysis of the above table that the proportion of memory occupied by the analysis method of the experimental group by the experimental group is much lower than the proportion of memory occupied by the comparison group analysis method by the operation platform.Calculate the average proportion of 
memory occupied by the two analysis methods during the experiment. The experimental group is $26.13 \%$, the comparison group is $50.81 \%$, and the comparison group is about 1.94 times the experimental group.It shows that the experimental group method can take up less memory to complete the same task. In summary, the performance of the GNSS signal-based wireless sensor network transmission delay analysis method studied in this paper is better.

\section{Concluding remarks}

The traditional network transmission delay analysis method occupies too much memory of the operating platform during operation, resulting in poor analysis performance. Therefore, this paper proposes a method for analyzing the transmission delay of wireless sensor networks based on GNSS signals. The comparison experiment with the traditional transmission delay analysis method proves that the analysis method studied in this paper takes up less memory and has better performance than the traditional method.

\section{Fund projects}

Research on Energy and Delay Optimization of Delay Sensitive Wireless Sensor Networks, Project No .: Xiangjiao Tong [2018] 519

\section{References}

[1] Wenyi, W., Fangping, Z.,Wanpeng, W., et al.: Real-time Estimation of Tropospheric Scattering Slant Delay of Low-elevation Obtained by Improved Ray Tracing.Journal of Electronics and Information Technology, Vol. 41, no. 10, pp. 2366-2372 (2019)

[2] Xuerui, W., Junming, X., Weihua, B., et al.: Theoretical analysis of soil freeze/thaw process on DDM waveform and multipath in order for GNSS-R/IR applications.Acta Geodaetica et Cartographica Sinica, Vol. 48, no. 08, pp. 1059-1066 (2019)

[3] Zhongpan, L.,Dongqing, Z., Minzhi, X., et al.: A Short-Time Fourier Transform Algorithm for Accelerating GNSS Signal Acquisition.Journal of Geomatics Science and Technology, Vol. 36, no. 01, pp. 23-27 (2019) 
[4]Gang, Z.,Lianbing, X.,Tianqi, Z.: NISI-MU-CDSK Communication System Based on Frequency Division Multiplexing.Journal of Shanghai Jiaotong University, Vol. 53, no. 05, pp. 575-583 (2019)

[5]Li, D., Qin, W.: Satellite Navigation Signal and Positioning Target Signal Delay Estimation.Computer Simulation, Vol. 36, no. 05, pp. 113-116 (2019)

[6] Yao, X., Xiaogang, R., Xiaoqing,Z.,et al.: Timestamp Delay Estimation for a Dynamic Exposure Monocular Visual-inertial System.Journal of Beijing University of Technology, Vol. 45, no. 06, pp. 515-523 (2019)

[7] Shuxin, J.,Zheng, Y.,Shenhui,J., et al.: High-Speed Non-Binary Coding and Modulation Techniques of GNSS Message.Journal of Beijing University of Posts and Telecommunications, Vol. 41, no. 04, pp. 97-103 (2018)

[8] Zhenglin, G., Zhengrong, L.,Junwei,N., et al.: Spoofing detection technique before despreading for GNSS antenna-array receivers.Journal of National University of Defense Technology, Vol. 40(02, pp. 91-96 (2018)

[9]Ping,W., Ziwen, S.: The Research of IWSN Real-Time Reliability Routing Based on Power Regulation.Chinese Journal of Sensors and Actuators, Vol. 31, no. 04, pp. 588-594 (2018)

[10] Jiaxin, C., Xinlong, W., Nengjie,Y., et al.: Modeling and intensity analysis of GNSS signal link for high-orbit spacecraft. Journal of Beijing University of Aeronautics and Astronautics, Vol. 44, no. 07, pp. 1496-1503 (2018)

[11] Junki, H., Hanshin, J., Cheol, M., et al.: Beamforming Based CSI Reference Signal Transmission for FDD Massive MIMO Systems. Journal of the Korean Institute of Communication Sciences, Vol. 41, no. 5, pp. 520-530 (2016)

[12] Esmail, Maged A., Ragheb, A,. Seleem, H., et al.: Radar signal transmission and switching over optical networks. Optics Communications, Vol. 410, pp. 385-388 (2018)

[13] Zhang, Q., Stojanovic, N., Prodaniuc, C., et al.: Single-lane 180 Gbit/s PAM-4 signal transmission over $2 \mathrm{~km}$ SSMF for short-reach applications. Optics Letters, Vol. 41, no. 19, pp. 4449-4452 (2016) 